\title{
Application of stochastic inequalities to global analysis of a nonlinear stochastic SIRS epidemic model with saturated treatment function
}

\author{
Shengqiang Zhang ${ }^{1}$, Xinzhu Meng ${ }^{1,2,3^{*}}$ (1) and Xinzeng Wang ${ }^{1}$
}

\author{
"Correspondence: \\ mxz0011@aliyun.com \\ ${ }^{1}$ College of Mathematics and \\ Systems Science, Shandong \\ University of Science and \\ Technology, Qingdao, P.R. China \\ ${ }^{2}$ State Key Laboratory of Mining \\ Disaster Prevention and Control \\ Co-founded by Shandong Province \\ and the Ministry of Science and \\ Technology, Shandong University of \\ Science and Technology, Qingdao, \\ P.R. China \\ Full list of author information is \\ available at the end of the article
}

\begin{abstract}
In this paper, we propose a new nonlinear stochastic SIRS epidemic model with standard incidence rate and saturated treatment function. The main purpose of this paper is to investigate the threshold dynamics of the nonlinear stochastic SIRS epidemic model by making use of stochastic inequality techniques. By using Lyapunov methods and Itô's formula, we first prove the existence and uniqueness of a global positive solution for the corresponding limiting system. Furthermore, we obtain sufficient conditions for the extinction and persistence in mean of the nonlinear stochastic SIRS epidemic model by using the techniques of a series of stochastic inequalities. Finally, we provide some numerical simulations to illustrate the performance of our theoretical findings.
\end{abstract}

Keywords: Stochastic SIRS epidemic model; Stochastic inequality; Saturated treatment; Standard incidence rate; Permanence in mean

\section{Introduction}

Mathematical inequalities play an important role in many fields of mathematical analysis and applications, especially for differential systems [1-5]. Recently, the inequalities techniques have been widely used to impulsive differential systems [6-10], stochastic differential systems [11-16] and impulsive stochastic differential systems [17-20], thus some new and interesting results have been obtained.

In the last few decades, infectious diseases have brought a series of troubles and great pain to millions of families. Many suitable measures are implemented to prevent the outbreak of infectious diseases. Various mathematical models are essential tools to study the spread of infectious diseases. Epidemiological models can analyze the underlying mechanisms which influence the expansion of infectious diseases. Let us assume that all individuals are divided into three compartments: $S(t)$ represents susceptible individuals who are susceptible to the disease; $I(t)$ represents infected individuals who are infected by the disease; and $R(t)$ represents recovered individuals who hold temporary immunity acquired from a disease, namely, after recovery, individuals lose immunity and move into the susceptible individuals. This is called SIRS model [21]. In most epidemic models, the bilinear

(c) The Author(s) 2018. This article is distributed under the terms of the Creative Commons Attribution 4.0 International License (http://creativecommons.org/licenses/by/4.0/), which permits unrestricted use, distribution, and reproduction in any medium, provided you give appropriate credit to the original author(s) and the source, provide a link to the Creative Commons license, and indicate if changes were made. 
incidence rate $\lambda S(t) I(t)$ is extensively used. Therefore, the dynamics of the SIRS epidemic model can be expressed by the following system of ordinary differential equations:

$$
\left\{\begin{array}{l}
\frac{\mathrm{d} S(t)}{\mathrm{d} t}=d N(t)-d S(t)-\lambda S(t) I(t)+v R(t) \\
\frac{\mathrm{d} I(t)}{\mathrm{d} t}=\lambda S(t) I(t)-(d+r) I(t) \\
\frac{\mathrm{d} R(t)}{\mathrm{d} t}=r I(t)-(d+v) R(t)
\end{array}\right.
$$

where the parameters $\lambda, d, v$ and $r$ are positive constants. Here, $N(t)=S(t)+I(t)+R(t)$ represents all individuals, $\lambda$ is the rate of transmission per contact, $d$ represents the diseased death rate and also represents the rate of recruitment of individuals, $v$ represents the rate at which recovered individuals lose immunity and return to the susceptible individuals, $r$ is the recovery rate of the infected individuals. Many researchers have studied several different SIRS epidemic models in the literature [21-25].

In epidemiological models, many different types of incidence rate play an important role. In 1986, Liu et al. [26] introduced a general incidence rate

$$
S f(I)=\frac{\lambda S I^{p}}{1+\alpha I^{q}}
$$

into the SIRS epidemic model. Here, $\lambda I^{p}$ is infection force of the disease and $\frac{1}{1+\alpha I^{q}}$ represents inhibition effect. The general incidence rate is more reasonable than the bilinear incidence rate because the general incidence rate takes into account the crowding effect and behavioral change of the infective individuals and prevents the unboundedness of the contact rate occurring by choosing suitable parameters. We notice that when $p=1$ and $\alpha=0$ or $q=0$, the general incidence rate (2) changes into the bilinear incidence rate. In recent years, a number of researchers [27-31] have investigated the nonlinear transmission laws more than the bilinear transmission laws. In 1992, Hethcote et al. [32] studied a standard incidence rate

$$
S f(I)=\frac{\lambda S I}{S+I+R}
$$

in the continuous-time SIRS epidemic model. In this paper, the authors analyzed the stability of the disease-free equilibrium and the endemic equilibrium for the SIRS model.

To prevent the spread of the infectious disease, many researchers [33-36] began to investigate a treatment function in the epidemic models. In 2004, Wang et al. [37] introduced a constant treatment function

$$
T(I)= \begin{cases}\beta, & I>0, \\ 0, & I=0\end{cases}
$$

into the SIR epidemic model. The authors found that to eradicate the disease, it is unnecessary to take such a large treatment capacity, and proved that disease spread may depend on a certain range of the initial parameters. In 2006, Wang [38] introduced a piecewise linear treatment function

$$
T(I)= \begin{cases}\beta I, & 0 \leq I \leq I_{0} \\ m, & I \geq I_{0}\end{cases}
$$


into the SIR model and proved that a backward bifurcation existed in this model. In 2011, Eckalbar et al. [39] adopted a quadratic treatment function

$$
T(I)=\max \left\{\beta I-g I^{2}, 0\right\}, \quad \beta, g>0
$$

into the SIR epidemic model and found four equilibria at most. Recently, the saturated treatment function has been frequently used in classical epidemic models. In 2008, Zhang et al. [40] introduced a saturated treatment function

$$
T(I)=\frac{\beta I}{1+\alpha I}
$$

into the SIR model, where $\beta>0, \alpha \geq 0$. $\beta$ is the cure rate and $\alpha$ represents the extent to which the infected effect delays the treatment. In [40], authors found that the valid methods for the control of disease were providing the patients timely treatment, enhancing the cure efficiency and decreasing the infective coefficient. According to (1), (3) and (4), in [41], Gao et al. took into account the SIRS epidemic model with standard incidence rate and saturated treatment function as follows:

$$
\left\{\begin{array}{l}
\frac{\mathrm{d} S(t)}{\mathrm{d} t}=\Lambda-d S(t)-\frac{\lambda S(t) I(t)}{N(t)}+v R(t) \\
\frac{\mathrm{d}(t)}{\mathrm{d} t}=\frac{\lambda S(t) I(t)}{N(t)}-(d+r) I(t)-\frac{\beta I(t)}{1+\alpha I(t)} \\
\frac{\mathrm{d} R(t)}{\mathrm{d} t}=r I(t)-(d+v) R(t)+\frac{\beta I(t)}{1+\alpha I(t)}
\end{array}\right.
$$

where $\Lambda$ is the recruitment rate of susceptible individuals, $d$ represents only the diseased death rate, $N(t) \equiv S(t)+I(t)+R(t)$ represents the total population size, other parameters have the same meaning as the ones above.

On the other hand, any systems are inevitably affected by various environmental noises, such as white noise, which are important components in an ecosystem. In 2001, May [42] showed that according to environmental fluctuation, the population birth and death rate, transmission coefficient and other parameters exhibit random perturbations to a lesser or greater extent. Consequently, we assume that the environmental fluctuation affects not only the standard incidence rate but also the saturated treatment rate of the disease, so that

$$
\lambda \rightarrow \lambda+\sigma_{1} \dot{B}_{1}(t), \quad \beta \rightarrow \beta+\sigma_{2} \dot{B}_{2}(t),
$$

where $B_{1}(t)$ and $B_{2}(t)$ represent standard Brownian motions with $B_{1}(0)=0$ and $B_{2}(0)=0$, $\sigma_{1}^{2}$ and $\sigma_{2}^{2}$ denote the intensities of white noise. In recent years, many researchers [14, 4352] have introduced stochastic environmental perturbations into deterministic population models to analyze the effects of environmental noise.

Motivated by the above work, we obtain the following stochastic SIRS epidemic model with standard incidence rate and saturated treatment function:

$$
\left\{\begin{array}{l}
\mathrm{d} S(t)=\left[\Lambda-d S(t)-\frac{\lambda S(t) I(t)}{N(t)}+v R(t)\right] \mathrm{d} t-\frac{\sigma_{1} S(t) I(t)}{N(t)} \mathrm{d} B_{1}(t), \\
\mathrm{d} I(t)=\left[\frac{\lambda S(t) I(t)}{N(t)}-(d+r) I(t)-\frac{\beta I(t)}{1+\alpha I(t)}\right] \mathrm{d} t+\frac{\sigma_{1} S(t) I(t)}{N(t)} \mathrm{d} B_{1}(t)-\frac{\sigma_{2} I(t)}{1+\alpha I(t)} \mathrm{d} B_{2}(t), \\
\mathrm{d} R(t)=\left[r I(t)-(d+v) R(t)+\frac{\beta I(t)}{1+\alpha I(t)}\right] \mathrm{d} t+\frac{\sigma_{2} I(t)}{1+\alpha I(t)} \mathrm{d} B_{2}(t) .
\end{array}\right.
$$


This paper is organized as follows. In Section 2, we first introduce preliminary knowledge and give some notations and lemmas. Furthermore, we prove the existence of a global positive solution for the corresponding limiting SIRS epidemic system. Moreover, we explore sufficient conditions for the persistence in mean and extinction of the stochastic SIRS epidemic system. In Section 3, we give a summary of the main results and a series of numerical simulations to illustrate the theoretical results.

\section{Main results}

The main purpose of our study is to investigate the threshold dynamics of the stochastic SIRS epidemic model which governs the extinction and permanence of the epidemic disease by applying the techniques of a series of stochastic inequalities.

\subsection{Preliminary knowledge}

In the section, we give some notations and lemmas which can be used for our main theoretical results. To this end, throughout this paper, unless otherwise specified, we let $\left(\Omega, \mathcal{F},\left\{\mathcal{F}_{t}\right\}_{t \geq 0}, \mathbb{P}\right)$ stand for a complete probability space with a filtration $\left\{\mathcal{F}_{t}\right\}_{t \geq 0}$ satisfying the usual conditions (i.e., it is increasing and right continuous while $\mathcal{F}_{0}$ contains all $\mathbb{P}$-null sets). For convenience, for an integrable function $f(t)$ on $\mathbb{R}_{+}=[0,+\infty)$, we define $\langle f(t)\rangle=\frac{1}{t} \int_{0}^{t} f(s) \mathrm{d} s$.

Generally speaking, an $n$-dimensional stochastic differential equation is expressed as follows:

$$
\mathrm{d} X(t)=F(t, X(t)) \mathrm{d} t+G(t, X(t)) \mathrm{d} B(t) .
$$

Here, $F(t, x)$ represents a function defined in $[0,+\infty) \times \mathbb{R}^{n}$ and $G(t, x)$ represents an $n \times$ $m$ matrix, $F(t, x)$ and $G(t, x)$ satisfy the locally Lipschitz conditions in $x . B(t)$ represents an $m$-dimensional standard Brownian motion defined on the complete probability space. $C^{2,1}\left(\mathbb{R}^{n} \times[0,+\infty), \mathbb{R}_{+}\right)$is a family of all nonnegative functions $V(x, t)$ which are defined on $\mathbb{R}^{n} \times[0,+\infty)$ such that this family of functions are continuously twice differentiable on $x$ and continuously once differentiable on $t$. In [53], Mao defined a differential operator $\mathcal{L}$ for the stochastic differential equation (6):

$$
\mathcal{L}=\frac{\partial}{\partial t}+\sum_{i=1}^{n} F_{i}(x, t) \frac{\partial}{\partial x_{i}}+\frac{1}{2} \sum_{i, j=1}^{n}\left[G^{T}(x, t) G(x, t)\right]_{i, j} \frac{\partial^{2}}{\partial x_{i} \partial x_{j}} .
$$

Applying $\mathcal{L}$ to a function $V(x, t) \in C^{2,1}\left(\mathbb{R}^{n} \times[0,+\infty), \mathbb{R}_{+}\right)$, we get

$$
\mathcal{L} V(x, t)=V_{t}(x, t)+V_{x}(x, t) F(x, t)+\frac{1}{2} \operatorname{trace}\left[G^{T}(x, t) V_{x x}(x, t) G(x, t)\right],
$$

where $V_{t}(x, t)=\frac{\partial V}{\partial t}, V_{x}(x, t)=\left(\frac{\partial V}{\partial x_{1}}, \frac{\partial V}{\partial x_{2}}, \ldots, \frac{\partial V}{\partial x_{n}}\right)$ and $V_{x x}(x, t)=\left(\frac{\partial^{2} V}{\partial x_{i} \partial x_{j}}\right)_{n \times n}$. By Itô's formula, when $x(t) \in \mathbb{R}^{n}$, we have

$$
\mathrm{d} V(x, t)=\mathcal{L} V(x, t) \mathrm{d} t+V_{x}(x, t) G(x, t) \mathrm{d} B(t) .
$$

Definition 2.1 ([54]) The definitions of extinction and persistence in mean are given as follows: 
(i) The population $X(t)$ is said to be extinct when $\lim _{t \rightarrow+\infty} X(t)=0$;

(ii) The population $X(t)$ is said to be persistent in mean when $\liminf _{t \rightarrow+\infty}\langle X(t)\rangle>m$, where $m$ is a positive constant.

The following inequalities can be used frequently in the sequel.

Lemma 2.1 (Burkholder-Davis-Gundy inequality [53]) Let $f(t) \in C^{2}\left(\mathbb{R}_{+} ; \mathbb{R}^{n \times m}\right)\left(C^{2}\left(\mathbb{R}_{+}\right.\right.$; $\left.\mathbb{R}^{n \times m}\right)$ be the family of processes $\{f(t)\}_{t \geq 0}$ such that, for every $T>0,\{f(t)\}_{0 \leq t \leq T} \in$ $C^{2}\left([0, T] ; \mathbb{R}^{n \times m}\right)$, where $C^{2}\left([0, T] ; \mathbb{R}^{n \times m}\right)$ represents the family of $R^{n \times m}$-valued $\mathcal{F}_{t}$-adapted processes $\{f(t)\}_{0 \leq t \leq T}$ such that $\left.\int_{0}^{T}|f(t)|^{2} d t<\infty\right)$. For every $t \geq 0$, we define

$$
M(t)=\int_{0}^{t} f(\theta) \mathrm{d} B(\theta), \quad A(t)=\int_{0}^{t}|f(\theta)|^{2} \mathrm{~d} \theta .
$$

Thus, for any $\eta>0$, there are two positive constants $c_{\eta}$ and $C_{\eta}$ such that

$$
c_{\eta} \mathbf{E}|A(t)|^{\frac{\eta}{2}} \leq \mathbf{E}\left(\sup _{0 \leq \theta \leq t}|M(\theta)|^{\eta}\right) \leq C_{\eta} \mathbf{E}|A(t)|^{\frac{\eta}{2}}, \quad t \geq 0
$$

where $c_{\eta}$ and $C_{\eta}$ only depend on $\eta$.

Lemma 2.2 (Chebyshev's inequality [53]) For all $c>0, \eta>0$ and $X \in L^{\eta}$, the following inequality holds:

$$
\mathbb{P}\{\xi:|X(\xi)| \geq c\} \leq \frac{\mathbf{E}|X|^{\eta}}{c^{\eta}}
$$

Lemma 2.3 (Doob's martingale inequality [53]) Let $X$ be a submartingale taking nonnegative real values, either in discrete or continuous time. That is, for all times $s$ and $t$ having $s<t$, we have

$$
X_{s} \leq \mathbf{E}\left[X_{t} \mid \mathcal{F}_{s}\right]
$$

Then, for any constant $c>0$,

$$
\mathbb{P}\left[\sup _{0 \leq t \leq T} X_{t} \geq c\right] \leq \frac{\mathbf{E}\left[\left|X_{T}\right|\right]}{c}
$$

where $\mathbb{P}$ denotes the probability measure on the sample space $\Omega$ of the stochastic process $X:[0, T] \times \Omega \rightarrow[0,+\infty]$ and $\mathbf{E}$ denotes the expected value with respect to the probability measure $\mathbb{P}$.

According to the biological meanings, we know that variables $S(t), I(t)$ and $R(t)$ of system (5) should be nonnegative when $t \geq 0$. Before we prove that the global positive solution of system (5) is existent and unique, we firstly investigate the following Lemma 2.4.

Lemma 2.4 For the positive solution $(S(t), I(t), R(t))$ of system (5) with any initial value $(S(0), I(0), R(0)) \in \mathbb{R}_{+}^{3}$, we have

$$
\max \left\{\limsup _{t \rightarrow+\infty} S(t), \limsup _{t \rightarrow+\infty} I(t), \limsup _{t \rightarrow+\infty} R(t)\right\} \leq \frac{\Lambda}{d}
$$


Proof For system (5), computing the sum of three equations yields

$$
\frac{\mathrm{d}(S(t)+I(t)+R(t))}{\mathrm{d} t}=\Lambda-d(S(t)+I(t)+R(t)) .
$$

Then we obtain

$$
\lim _{t \rightarrow+\infty}(S(t)+I(t)+R(t))=\frac{\Lambda}{d}
$$

It is easy to see that

$$
\limsup _{t \rightarrow+\infty} S(t) \leq \frac{\Lambda}{d}, \quad \limsup _{t \rightarrow+\infty} I(t) \leq \frac{\Lambda}{d}, \quad \limsup _{t \rightarrow+\infty} R(t) \leq \frac{\Lambda}{d},
$$

and $S(t) \geq 0, I(t) \geq 0, R(t) \geq 0$.

This completes the proof of Lemma 2.4.

According to Lemma 2.4, we can know that at any equilibrium $E^{*}=\left(S^{*}, I^{*}, R^{*}\right)$ of system (5), $N^{*} \equiv S^{*}+I^{*}+R^{*} \equiv \frac{\Lambda}{d}$, then

$$
\Omega=\left\{(S(t), I(t), R(t)): S(t) \geq 0, I(t) \geq 0, R(t) \geq 0, S(t)+I(t)+R(t) \equiv \frac{\Lambda}{d}\right\}
$$

is a positively invariant region for the system. Therefore, we only consider the dynamics of system (5) in $\Omega$.

The set $\Omega$ is a positive invariant region for system (5), thus we assume the population size has reached limiting value; in other words, $N(t) \equiv \frac{\Lambda}{d} \equiv S(t)+I(t)+R(t)$, and we obtain $R(t) \equiv \frac{\Lambda}{d}-S(t)-I(t)$. Obviously, system (5) can be reduced to the following system:

$$
\left\{\begin{array}{l}
\mathrm{d} S(t)=\left[\Lambda-d S(t)-\frac{\lambda d S(t) I(t)}{\Lambda}+v\left(\frac{\Lambda}{d}-S(t)-I(t)\right)\right] \mathrm{d} t-\frac{d \sigma_{1} S(t) I(t)}{\Lambda} \mathrm{d} B_{1}(t), \\
\mathrm{d} I(t)=\left[\frac{\lambda d S(t) I(t)}{\Lambda}-(d+r) I(t)-\frac{\beta I(t)}{1+\alpha I(t)}\right] \mathrm{d} t+\frac{d \sigma_{1} S(t) I(t)}{\Lambda} \mathrm{d} B_{1}(t)-\frac{\sigma_{2} I(t)}{1+\alpha I(t)} \mathrm{d} B_{2}(t) .
\end{array}\right.
$$

Therefore, system (7) is equivalent to system (5). Next, we prove the existence and uniqueness of the global positive solution of system (7).

Lemma 2.5 For any given initial value $(S(0), I(0)) \in \mathbb{R}_{+}^{2}$, model (7) has a unique positive solution $(S(t), I(t))$ on $t \geq 0$, and the solution remains in $\mathbb{R}_{+}^{2}$ with probability 1 , namely $(S(t), I(t)) \in \mathbb{R}_{+}^{2}$ for all $t \geq 0$ almost surely.

Proof Since all the coefficients of system (7) satisfy the local Lipschitz condition, for any initial value $(S(0), I(0)) \in \mathbb{R}_{+}^{2}$, there exists a unique local solution $(S(t), I(t))$ on $\left[0, \tau_{e}\right)$, where $\tau_{e}$ represents the explosion time. Then $\tau_{e}=+\infty$ demonstrates that the solution of system (7) is global. Therefore, we let $k_{0} \geq 1$ be a sufficiently large constant for every component of $(S(0), I(0))$ all lying within the interval $\left[\frac{1}{k_{0}}, k_{0}\right] \times\left[\frac{1}{k_{0}}, k_{0}\right]$. For each integer $k>k_{0}$, we define the stopping time as follows:

$$
\tau_{k}=\inf \left\{t \in\left[0, \tau_{e}\right): \min \{(S(t), I(t))\} \leq \frac{1}{k} \text { or } \max \{(S(t), I(t))\} \geq k\right\} .
$$


Throughout this paper, we let $\inf \emptyset=\infty$ (as usual $\emptyset$ denotes the empty set). It is easy to see that $\tau_{k}$ is increasing as $k \rightarrow+\infty$. We set $\tau_{\infty}=\lim \sup _{k \rightarrow+\infty} \tau_{k}$, obviously $\tau_{\infty} \leq \tau_{e}$ a.s. If we can show that $\tau_{\infty}=+\infty$ a.s., then we can obtain that $\tau_{e}=+\infty$. Next, we only need to show $\tau_{\infty}=+\infty$ a.s. Assuming this assertion is not true, there exist a constant $T>0$ and $\varepsilon \in(0,1)$ such that $\mathbb{P}\left\{\tau_{\infty} \leq T\right\}>\varepsilon$. Thus, there exists an integer $k_{1} \geq k_{0}$ such that

$$
\mathbb{P}\left\{\tau_{k} \leq T\right\}>\varepsilon \quad \text { for all } k \geq k_{1} .
$$

We define a $C^{2}$-function $V: \mathbb{R}_{+}^{2} \rightarrow \mathbb{R}_{+}$by

$$
V(S(t), I(t))=(S(t)-1-\ln S(t))+(I(t)-1-\ln I(t)) .
$$

Applying Itô’s formula leads to

$$
\mathrm{d} V(S(t), I(t))=\mathcal{L} V(S(t), I(t)) \mathrm{d} t+\frac{d \sigma_{1}(I(t)-S(t))}{\Lambda} \mathrm{d} B_{1}(t)-\frac{\sigma_{2}(I(t)-1)}{1+\alpha I(t)} \mathrm{d} B_{2}(t),
$$

where

$$
\begin{aligned}
\mathcal{L} V(S(t), I(t))= & \left(1-\frac{1}{S(t)}\right)\left[\Lambda-d S(t)-\frac{\lambda d S(t) I(t)}{\Lambda}+v\left(\frac{\Lambda}{d}-S(t)-I(t)\right)\right] \\
& +\left(1-\frac{1}{I(t)}\right)\left[\frac{\lambda d S(t) I(t)}{\Lambda}-(d+r) I(t)-\frac{\beta I(t)}{1+\alpha I(t)}\right] \\
& +\frac{1}{2}\left[\frac{d^{2} \sigma_{1}^{2} I^{2}(t)}{\Lambda^{2}}+\frac{d^{2} \sigma_{1}^{2} S^{2}(t)}{\Lambda^{2}}+\frac{\sigma_{2}^{2}}{(1+\alpha I(t))^{2}}\right] \\
= & \Lambda-d S(t)-\frac{\lambda d S(t) I(t)}{\Lambda}+v\left(\frac{\Lambda}{d}-S(t)-I(t)\right)-\frac{\Lambda}{S(t)}+d+\frac{\lambda d I(t)}{\Lambda} \\
& -\frac{v\left(\frac{\Lambda}{d}-S(t)-I(t)\right)}{S(t)}+\frac{\lambda d S(t) I(t)}{\Lambda}-(d+r) I(t)-\frac{\beta I(t)}{1+\alpha I(t)}-\frac{\lambda d S(t)}{\Lambda} \\
& +d+r+\frac{\beta}{1+\alpha I(t)}+\frac{d^{2} \sigma_{1}^{2} I^{2}(t)}{2 \Lambda^{2}}+\frac{d^{2} \sigma_{1}^{2} S^{2}(t)}{2 \Lambda^{2}}+\frac{\sigma_{2}^{2}}{2(1+\alpha I(t))^{2}} \\
\leq & \Lambda+\frac{v \Lambda}{d}+2 d+\lambda+r+\beta+\sigma_{1}^{2}+\frac{\sigma_{2}^{2}}{2} \\
\leq & K,
\end{aligned}
$$

where $K$ is a positive constant which is independent of $S(t), I(t)$ and $t$.

Therefore, we can have

$$
\mathrm{d} V(S(t), I(t)) \leq K \mathrm{~d} t+\frac{d \sigma_{1}(I(t)-S(t))}{\Lambda} \mathrm{d} B_{1}(t)-\frac{\sigma_{2}(I(t)-1)}{1+\alpha I(t)} \mathrm{d} B_{2}(t) .
$$

Integrating (9) from 0 to $T \wedge \tau_{k}=\min \left\{T, \tau_{k}\right\}$ and taking expectation on both sides yield

$$
\mathbf{E} V\left(S\left(T \wedge \tau_{k}\right), I\left(T \wedge \tau_{k}\right)\right) \leq V(S(0), I(0))+K \mathbf{E}\left(T \wedge \tau_{k}\right)
$$

then

$$
\mathbf{E} V\left(S\left(T \wedge \tau_{k}\right), I\left(T \wedge \tau_{k}\right)\right) \leq V(S(0), I(0))+K T .
$$


Set $\Omega_{k}=\left\{\tau_{k} \leq T\right\}$, from inequality (8), we can know that $\mathbb{P}\left(\Omega_{k}\right) \geq \varepsilon$. And for all $\omega \in \Omega_{k}$, there exists at least one of $S\left(\tau_{k}, \omega\right), I\left(\tau_{k}, \omega\right)$ which equals either $k$ or $\frac{1}{k}$. Thus, we get $V\left(S\left(\tau_{k}, \omega\right), I\left(\tau_{k}, \omega\right)\right)$ is no less than either

$$
k-1-\ln k \text { or } \frac{1}{k}-1-\ln \frac{1}{k}=\frac{1}{k}-1+\ln k .
$$

As a result, we can have

$$
V\left(S\left(T \wedge \tau_{k}\right), I\left(T \wedge \tau_{k}\right)\right) \geq(k-1-\ln k) \wedge\left(\frac{1}{k}-1+\ln k\right) .
$$

Combining equations (10) and (11), we can have

$$
\begin{aligned}
V(S(0), I(0))+K T & \geq \mathbf{E}\left[1 \Omega_{k}(\omega) V\left(S\left(T \wedge \tau_{k}\right), I\left(T \wedge \tau_{k}\right)\right)\right] \\
& \geq \varepsilon(k-1-\ln k) \wedge\left(\frac{1}{k}-1+\ln k\right),
\end{aligned}
$$

where $1 \Omega_{k}$ denotes the indicator function of $\Omega_{k}$. When $k \rightarrow+\infty$, we obtain

$$
+\infty>V(S(0), I(0))+K T=+\infty
$$

which is a contradiction. Thus, we obtain that $\tau_{\infty}=+\infty$ a.s.

This completes the proof of Lemma 2.5 .

Lemma 2.6 Let $(S(t), I(t))$ be a solution of system (7) with any initial value $(S(0), I(0)) \in \mathbb{R}_{+}^{2}$, then

$$
\begin{array}{ll}
\lim _{t \rightarrow+\infty} \frac{1}{t} \int_{0}^{t} \frac{d \sigma_{1} S(\theta)}{\Lambda} \mathrm{d} B_{1}(\theta)=0, & \lim _{t \rightarrow+\infty} \frac{1}{t} \int_{0}^{t} \frac{\sigma_{2}}{1+\alpha I(\theta)} \mathrm{d} B_{2}(\theta)=0, \\
\lim _{t \rightarrow+\infty} \frac{1}{t} \int_{0}^{t} \frac{\sigma_{2} I(\theta)}{1+\alpha I(\theta)} \mathrm{d} B_{2}(\theta)=0, & \lim _{t \rightarrow+\infty} \frac{1}{t} \int_{0}^{t} \frac{\sigma_{2}}{\alpha} \mathrm{d} B_{1}(\theta)=0 .
\end{array}
$$

Proof We let $M_{1}(t)=\int_{0}^{t} \frac{d \sigma_{1} S(\theta)}{\Lambda} \mathrm{d} B_{1}(\theta)$ and $\eta>2$. Making use of Lemma 2.1 for the Burkholder-Davis-Gundy inequality and the results of Lemma 2.4, we can get

$$
\begin{aligned}
\mathbf{E}\left[\sup _{0 \leq \theta \leq t}\left|M_{1}(\theta)\right|^{\eta}\right] & \leq C_{\eta} \mathbf{E}\left[\int_{0}^{t} \frac{d^{2} \sigma_{1}^{2} S^{2}(\theta)}{\Lambda^{2}} \mathrm{~d} \theta\right]^{\frac{\eta}{2}} \leq t^{\frac{\eta}{2}} C_{\eta} \mathbf{E}\left[\sup _{0 \leq \theta \leq t} \frac{d^{\eta} \sigma_{1}^{\eta} S^{\eta}(\theta)}{\Lambda^{\eta}}\right] \\
& \leq t^{\frac{\eta}{2}} C_{\eta} \sigma_{1}^{\eta} .
\end{aligned}
$$

Here, we set $\epsilon$ be an arbitrary positive constant. Thus, applying Lemma 2.2 for Chebyshev's inequality yields

$$
\begin{aligned}
\mathbb{P}\left\{\xi: \sup _{k \delta \leq t \leq(k+1) \delta}\left|M_{1}(t)\right|^{\eta}>(k \delta)^{1+\epsilon+\frac{\eta}{2}}\right\} & \leq \frac{\mathbf{E}\left(\left|M_{1}((k+1) \delta)\right|^{\eta}\right)}{(k \delta)^{1+\epsilon+\frac{\eta}{2}}} \leq \frac{\sigma_{1}^{\eta} C_{\eta}[(1+k) \delta]^{\frac{\eta}{2}}}{(k \delta)^{1+\epsilon+\frac{\eta}{2}}} \\
& \leq \frac{2^{\frac{\eta}{2}} \sigma_{1}^{\eta} C_{\eta}}{(k \delta)^{1+\epsilon}} .
\end{aligned}
$$


Using Lemma 2.3 for Doob's martingale inequality and the Borel-Cantelli lemma in [53], for almost every $\xi \in \Omega$, we always get that

$$
\sup _{k \delta \leq t \leq(k+1) \delta}\left|M_{1}(t)\right|^{\eta} \leq(k \delta)^{1+\epsilon+\frac{\eta}{2}}
$$

satisfies for all except finitely some $k$. Thus, there is a positive number $k_{0}(\xi)$, for almost every $\xi \in \Omega$, inequality (12) holds when $k \geq k_{0}(\xi)$. Therefore, when both $k \geq k_{0}(\xi)$ and $k \delta \leq t \leq(k+1) \delta$ hold, for almost all $\xi \in \Omega$, we get

$$
\frac{\ln \left|M_{1}(t)\right|^{\eta}}{\ln t} \leq \frac{\left(1+\epsilon+\frac{\eta}{2}\right) \ln (k \delta)}{\ln (k \delta)}=1+\epsilon+\frac{\eta}{2}
$$

Thus, it is easy to see that

$$
\limsup _{t \rightarrow+\infty} \frac{\ln \left|M_{1}(t)\right|}{\ln t} \leq \frac{1+\epsilon+\frac{\eta}{2}}{\eta}
$$

Let $\epsilon \rightarrow 0$, we can have that

$$
\limsup _{t \rightarrow+\infty} \frac{\ln \left|M_{1}(t)\right|}{\ln t} \leq \frac{1}{2}+\frac{1}{\eta} \text { a.s. }
$$

So, for an arbitrary small positive constant $\zeta\left(\zeta<\frac{1}{2}-\frac{1}{\eta}\right)$, there is a constant $T(\xi)$ and a set $\Omega_{\zeta}$ such that $\mathbb{P}\left(\Omega_{\zeta}\right) \geq 1-\zeta$. Furthermore, for $t \geq T(\xi), \xi \in \Omega_{\zeta}$, we have

$$
\ln \left|M_{1}(t)\right| \leq\left(\frac{1}{2}+\frac{1}{\eta}+\zeta\right) \ln t
$$

As a result, we get

$$
\limsup _{t \rightarrow+\infty} \frac{M_{1}(t)}{t} \leq \limsup _{t \rightarrow+\infty} \frac{t^{\frac{1}{2}+\frac{1}{\eta}+\zeta}}{t}=0 \quad \text { a.s. }
$$

On the other hand, we know

$$
\limsup _{t \rightarrow+\infty} \frac{\left|M_{1}(t)\right|}{t} \geq 0 \quad \text { a.s. }
$$

To sum up

$$
\lim _{t \rightarrow+\infty} \frac{\left|M_{1}(t)\right|}{t}=0 \quad \text { a.s. }
$$

That is to say,

$$
\lim _{t \rightarrow+\infty} \frac{M_{1}(t)}{t}=\lim _{t \rightarrow+\infty} \frac{1}{t} \int_{0}^{t} \frac{d \sigma_{1} S(\theta)}{\Lambda} \mathrm{d} B_{1}(\theta)=0 \quad \text { a.s. }
$$

Making use of the same argument, we let $M_{2}(t)=\int_{0}^{t} \frac{\sigma_{2}}{1+\alpha I(\theta)} \mathrm{d} B_{2}(\theta)$, then we can get

$$
\limsup _{t \rightarrow+\infty} \frac{\left\langle M_{2}, M_{2}\right\rangle_{t}}{t} \leq \limsup _{t \rightarrow+\infty} \frac{1}{t} \int_{0}^{t} \sigma_{2}^{2} \mathrm{~d} \theta=\sigma_{2}^{2}<+\infty \quad \text { a.s. }
$$


Using the strong law of large numbers, we can obtain

$$
\lim _{t \rightarrow+\infty} \frac{M_{2}(t)}{t}=0 \quad \text { a.s. }
$$

i.e.,

$$
\lim _{t \rightarrow+\infty} \frac{M_{2}(t)}{t}=\lim _{t \rightarrow+\infty} \frac{1}{t} \int_{0}^{t} \frac{\sigma_{2}}{1+\alpha I(\theta)} \mathrm{d} B_{2}(\theta)=0 \quad \text { a.s. }
$$

Let $M_{3}(t)=\int_{0}^{t} \frac{\sigma_{2} I(\theta)}{1+\alpha I(\theta)} \mathrm{d} B_{2}(\theta)$, then we can get

$$
\limsup _{t \rightarrow+\infty} \frac{\left\langle M_{3}, M_{3}\right\rangle_{t}}{t} \leq \limsup _{t \rightarrow+\infty} \frac{1}{t} \int_{0}^{t} \frac{\sigma_{2}^{2}}{\alpha^{2}} \mathrm{~d} \theta=\frac{\sigma_{2}^{2}}{\alpha^{2}}<+\infty \quad \text { a.s. }
$$

Using the strong law of large numbers, we can obtain

$$
\lim _{t \rightarrow+\infty} \frac{M_{3}(t)}{t}=0 \quad \text { a.s. }
$$

i.e.,

$$
\lim _{t \rightarrow+\infty} \frac{M_{3}(t)}{t}=\lim _{t \rightarrow+\infty} \frac{1}{t} \int_{0}^{t} \frac{\sigma_{2} I(\theta)}{1+\alpha I(\theta)} \mathrm{d} B_{2}(\theta)=0 \quad \text { a.s. }
$$

Let $M_{4}(t)=\int_{0}^{t} \frac{\sigma_{2}}{\alpha} \mathrm{d} B_{2}(\theta)$, then we can get

$$
\limsup _{t \rightarrow+\infty} \frac{\left\langle M_{4}, M_{4}\right\rangle_{t}}{t}=\limsup _{t \rightarrow+\infty} \frac{1}{t} \int_{0}^{t} \frac{\sigma_{2}^{2}}{\alpha^{2}} \mathrm{~d} \theta=\frac{\sigma_{2}^{2}}{\alpha^{2}}<+\infty \quad \text { a.s. }
$$

Using the strong law of large numbers, we can obtain

$$
\lim _{t \rightarrow+\infty} \frac{M_{4}(t)}{t}=0 \quad \text { a.s. }
$$

i.e.,

$$
\lim _{t \rightarrow+\infty} \frac{M_{4}(t)}{t}=\lim _{t \rightarrow+\infty} \frac{1}{t} \int_{0}^{t} \frac{\sigma_{2}}{\alpha} \mathrm{d} B_{2}(\theta)=0 \quad \text { a.s. }
$$

The proof of Lemma 2.6 is complete.

\subsection{Extinction}

When we study an epidemic model, extinction and persistence in mean are two important properties. In this subsection, we explore the conditions which lead to the extinction of epidemic system (7) under stochastic disturbances.

Next, we explore the conditions for the extinction of the epidemic disease of stochastic system (7).

Theorem 2.1 Let $(S(t), I(t))$ be the solution of system (7) with any initial value $(S(0), I(0)) \in$ $\mathbb{R}_{+}^{2}$. When one of the following conditions holds: 
(i) $R_{1}=\frac{\lambda^{2}}{2(d+r) \sigma_{1}^{2}}+\frac{\beta^{2}}{2(d+r) \sigma_{2}^{2}}<1$,

(ii) $\lambda \geq \sigma_{1}^{2}$ and $R_{2}=\frac{2(\lambda-d-r)(d+\alpha \Lambda)^{2}}{\sigma_{1}^{2}(d+\alpha \Lambda)^{2}+d^{2} \sigma_{2}^{2}}-\frac{2 d \beta(d+\alpha \Lambda)}{\sigma_{1}^{2}(d+\alpha \Lambda)^{2}+d^{2} \sigma_{2}^{2}}<1$,

(iii) $\lambda<\sigma_{1}^{2}$ and $R_{3}=\frac{\lambda^{2}(d+\alpha \Lambda)}{2 \sigma_{1}^{2}[d \beta+(d+r)(d+\alpha \Lambda)]}-\frac{d^{2} \sigma_{2}^{2}}{2\left[d \beta(d+\alpha \Lambda)+(d+r)(d+\alpha \Lambda)^{2}\right]}<1$,

(iv) $\lambda \geq \sigma_{1}^{2}$ and $R_{4}=\frac{2 \lambda}{\sigma_{1}^{2}+2(d+r)}+\frac{\beta^{2}}{\sigma_{2}^{2}\left[\sigma_{1}^{2}+2(d+r)\right]}<1$,

then

$$
\lim _{t \rightarrow+\infty} S(t)=\frac{\Lambda}{d}, \quad \lim _{t \rightarrow+\infty} I(t)=0 .
$$

Proof Making use of Itô's formula to the second equation of the stochastic differential system (7), we get

$$
\begin{aligned}
\mathrm{d} \ln I(t)= & \frac{1}{I(t)}\left[\left(\frac{\lambda d S(t) I(t)}{\Lambda}-(d+r) I(t)-\frac{\beta I(t)}{1+\alpha I(t)}\right) \mathrm{d} t+\frac{d \sigma_{1} S(t) I(t)}{\Lambda} \mathrm{d} B_{1}(t)\right. \\
& \left.-\frac{\sigma_{2} I(t)}{1+\alpha I(t)} \mathrm{d} B_{2}(t)\right]+\frac{1}{2}\left(-\frac{1}{I^{2}(t)}\right)\left[\frac{d^{2} \sigma_{1}^{2} S^{2}(t) I^{2}(t)}{\Lambda^{2}}+\frac{\sigma_{2}^{2} I^{2}(t)}{(1+\alpha I(t))^{2}}\right] \mathrm{d} t \\
= & \left(\frac{\lambda d S(t)}{\Lambda}-(d+r)-\frac{\beta}{1+\alpha I(t)}-\frac{d^{2} \sigma_{1}^{2} S^{2}(t)}{2 \Lambda^{2}}-\frac{\sigma_{2}^{2}}{2(1+\alpha I(t))^{2}}\right) \mathrm{d} t \\
& +\frac{d \sigma_{1} S(t)}{\Lambda} \mathrm{d} B_{1}(t)-\frac{\sigma_{2}}{1+\alpha I(t)} \mathrm{d} B_{2}(t) \\
= & -\frac{d^{2} \sigma_{1}^{2}}{2 \Lambda^{2}}\left(S^{2}(t)-\frac{2 \lambda \Lambda}{d \sigma_{1}^{2}} S(t)\right) \\
& \left.-\frac{\sigma_{2}^{2}}{2}\left(\frac{1}{(1+\alpha I(t))^{2}}+\frac{2 \beta}{\sigma_{2}^{2}(1+\alpha I(t))}\right)-(d+r)\right] \mathrm{d} t \\
& +\frac{d \sigma_{1} S(t)}{\Lambda} \mathrm{d} B_{1}(t)-\frac{\sigma_{2}}{1+\alpha I(t)} \mathrm{d} B_{2}(t) \\
& +\frac{d \sigma_{1} S(t)}{\Lambda} \mathrm{d} B_{1}(t)-\frac{\sigma_{2}}{1+\alpha I(t)} \mathrm{d} B_{2}(t) . \\
= & \left.-\frac{d^{2} \sigma_{1}^{2}}{2 \Lambda^{2}}\left(S(t)-\frac{\lambda \Lambda}{d \sigma_{1}^{2}}\right)^{2}-\frac{\sigma_{2}^{2}}{2}\left(\frac{1}{1+\alpha I(t)}+\frac{\beta}{\sigma_{2}^{2}}\right)^{2}+\frac{\lambda^{2}}{2 \sigma_{1}^{2}}+\frac{\beta^{2}}{2 \sigma_{2}^{2}}-(d+r)\right] \mathrm{d} t \\
& (13) \\
& \\
&
\end{aligned}
$$

Case (i): When $R_{1}=\frac{\lambda^{2}}{2(d+r) \sigma_{1}^{2}}+\frac{\beta^{2}}{2(d+r) \sigma_{2}^{2}}<1$ holds, integrating both sides of inequality (13) from 0 to $t$ gives

$$
\begin{aligned}
\ln I(t)= & -\frac{d^{2} \sigma_{1}^{2}}{2 \Lambda^{2}} \int_{0}^{t}\left(S(\theta)-\frac{\lambda \Lambda}{d \sigma_{1}^{2}}\right)^{2} \mathrm{~d} \theta-\frac{\sigma_{2}^{2}}{2} \int_{0}^{t}\left(\frac{1}{1+\alpha I(\theta)}+\frac{\beta}{\sigma_{2}^{2}}\right)^{2} \mathrm{~d} \theta \\
& -\left(d+r-\frac{\lambda^{2}}{2 \sigma_{1}^{2}}-\frac{\beta^{2}}{2 \sigma_{2}^{2}}\right) t+M_{1}(t)-M_{2}(t)+\ln I(0) \\
\leq & -\left(d+r-\frac{\lambda^{2}}{2 \sigma_{1}^{2}}-\frac{\beta^{2}}{2 \sigma_{2}^{2}}\right) t+M_{1}(t)-M_{2}(t)+\ln I(0)
\end{aligned}
$$

where $M_{1}(t)=\int_{0}^{t} \frac{d \sigma_{1} S(\theta)}{\Lambda} \mathrm{d} B_{1}(\theta)$ and $M_{2}(t)=\int_{0}^{t} \frac{\sigma_{2}}{1+\alpha I(\theta)} \mathrm{d} B_{2}(\theta)$.

Dividing both sides of inequality (14) by $t$ yields

$$
\frac{\ln I(t)}{t} \leq-\left(d+r-\frac{\lambda^{2}}{2 \sigma_{1}^{2}}-\frac{\beta^{2}}{2 \sigma_{2}^{2}}\right)+\frac{M_{1}(t)}{t}-\frac{M_{2}(t)}{t}+\frac{\ln I(0)}{t}
$$


The functions $M_{1}(t)$ and $M_{2}(t)$ are known as the local continuous martingale with $M_{1}(0)=$ 0 and $M_{2}(0)=0$. Applying Lemma 2.6, we can know

$$
\lim _{t \rightarrow+\infty} \frac{M_{1}(t)}{t}=0, \quad \lim _{t \rightarrow+\infty} \frac{M_{2}(t)}{t}=0 \quad \text { a.s. }
$$

Making use of the condition $R_{1}=\frac{\lambda^{2}}{2(d+r) \sigma_{1}^{2}}+\frac{\beta^{2}}{2(d+r) \sigma_{2}^{2}}<1$ and taking the limit superior of both sides of inequality (15), we can get

$$
\limsup _{t \rightarrow+\infty} \frac{\ln I(t)}{t} \leq-\left(d+r-\frac{\lambda^{2}}{2 \sigma_{1}^{2}}-\frac{\beta^{2}}{2 \sigma_{2}^{2}}\right)=(d+r)\left(R_{1}-1\right)<0,
$$

which implies $\lim _{t \rightarrow+\infty} I(t)=0$.

Case (ii): When both $\lambda \geq \sigma_{1}^{2}$ and $R_{2}=\frac{2(\lambda-d-r)(d+\alpha \Lambda)^{2}}{\sigma_{1}^{2}(d+\alpha \Lambda)^{2}+d^{2} \sigma_{2}^{2}}-\frac{2 d \beta(d+\alpha \Lambda)}{\sigma_{1}^{2}(d+\alpha \Lambda)^{2}+d^{2} \sigma_{2}^{2}}<1$ hold, according to inequality (13), we can get

$$
\begin{aligned}
\mathrm{d} \ln I(t) \leq & {\left[-\frac{d^{2} \sigma_{1}^{2}}{2 \Lambda^{2}}\left(S(t)-\frac{\lambda \Lambda}{d \sigma_{1}^{2}}\right)^{2}+\frac{\lambda^{2}}{2 \sigma_{1}^{2}}-\frac{d^{2} \sigma_{2}^{2}}{2(d+\alpha \Lambda)^{2}}-\frac{d \beta}{d+\alpha \Lambda}-(d+r)\right] \mathrm{d} t } \\
& +\frac{d \sigma_{1} S(t)}{\Lambda} \mathrm{d} B_{1}(t)-\frac{\sigma_{2}}{1+\alpha I(t)} \mathrm{d} B_{2}(t) .
\end{aligned}
$$

Since $\lambda \geq \sigma_{1}^{2}$, the variable $S(t)$ in inequality (16) takes its maximum value on the interval $\left[0, \frac{\Lambda}{d}\right]$ at $\frac{\Lambda}{d}$, thus we have

$$
\begin{aligned}
\mathrm{d} \ln I(t) \leq & {\left[-\frac{\sigma_{1}^{2}}{2}+\lambda-\frac{d^{2} \sigma_{2}^{2}}{2(d+\alpha \Lambda)^{2}}-\frac{d \beta}{d+\alpha \Lambda}-(d+r)\right] \mathrm{d} t } \\
& +\frac{d \sigma_{1} S(t)}{\Lambda} \mathrm{d} B_{1}(t)-\frac{\sigma_{2}}{1+\alpha I(t)} \mathrm{d} B_{2}(t) .
\end{aligned}
$$

Integrating both sides of inequality (17) from 0 to $t$ and dividing both sides by $t$, we obtain

$$
\begin{aligned}
\frac{\ln I(t)}{t} \leq & {\left[-\frac{\sigma_{1}^{2}}{2}+\lambda-\frac{d^{2} \sigma_{2}^{2}}{2(d+\alpha \Lambda)^{2}}-\frac{d \beta}{d+\alpha \Lambda}-(d+r)\right] } \\
& +\frac{M_{1}(t)}{t}-\frac{M_{2}(t)}{t}+\frac{\ln I(0)}{t} .
\end{aligned}
$$

Using the condition $R_{2}=\frac{2(\lambda-d-r)(d+\alpha \Lambda)^{2}}{\sigma_{1}^{2}(d+\alpha \Lambda)^{2}+d^{2} \sigma_{2}^{2}}-\frac{2 d \beta(d+\alpha \Lambda)}{\sigma_{1}^{2}(d+\alpha \Lambda)^{2}+d^{2} \sigma_{2}^{2}}<1$, by Lemma 2.6 and taking the limit superior of both sides of inequality (18), one has

$$
\begin{aligned}
\limsup _{t \rightarrow+\infty} \frac{\ln I(t)}{t} & \leq-\left[\left(\frac{\sigma_{1}^{2}}{2}+\frac{d^{2} \sigma_{2}^{2}}{2(d+\alpha \Lambda)^{2}}\right)-\left(\lambda-\frac{d \beta}{d+\alpha \Lambda}-d-r\right)\right] \\
& =\frac{1}{2}\left(\sigma_{1}^{2}+\frac{d^{2} \sigma_{2}^{2}}{(d+\alpha \Lambda)^{2}}\right)\left(R_{2}-1\right)<0,
\end{aligned}
$$

which implies $\lim _{t \rightarrow+\infty} I(t)=0$.

Case (iii): When both $\lambda<\sigma_{1}^{2}$ and $R_{3}=\frac{\lambda^{2}(d+\alpha \Lambda)}{2 \sigma_{1}^{2}[d \beta+(d+r)(d+\alpha \Lambda)]}-\frac{d^{2} \sigma_{2}^{2}}{2\left[d \beta(d+\alpha \Lambda)+(d+r)(d+\alpha \Lambda)^{2}\right]}<1$ hold, since $\lambda<\sigma_{1}^{2}$, the variable $S(t)$ in inequality (16) takes its maximum value on the interval 
$\left[0, \frac{\Lambda}{d}\right]$ at $\frac{\lambda \Lambda}{d \sigma_{1}^{2}}$, so we have

$$
\begin{aligned}
\mathrm{d} \ln I(t) \leq & {\left[\frac{\lambda^{2}}{2 \sigma_{1}^{2}}-\frac{d^{2} \sigma_{2}^{2}}{2(d+\alpha \Lambda)^{2}}-\frac{d \beta}{d+\alpha \Lambda}-(d+r)\right] \mathrm{d} t } \\
& +\frac{d \sigma_{1} S(t)}{\Lambda} \mathrm{d} B_{1}(t)-\frac{\sigma_{2}}{1+\alpha I(t)} \mathrm{d} B_{2}(t) .
\end{aligned}
$$

Integrating both sides of inequality (19) from 0 to $t$ and dividing both sides by $t$, we can obtain

$$
\frac{\ln I(t)}{t} \leq\left[\frac{\lambda^{2}}{2 \sigma_{1}^{2}}-\frac{d^{2} \sigma_{2}^{2}}{2(d+\alpha \Lambda)^{2}}-\frac{d \beta}{d+\alpha \Lambda}-(d+r)\right]+\frac{M_{1}(t)}{t}-\frac{M_{2}(t)}{t}+\frac{\ln I(0)}{t} .
$$

Applying the condition $R_{3}=\frac{\lambda^{2}(d+\alpha \Lambda)}{2 \sigma_{1}^{2}[d \beta+(d+r)(d+\alpha \Lambda)]}-\frac{d^{2} \sigma_{2}^{2}}{2\left[d \beta(d+\alpha \Lambda)+(d+r)(d+\alpha \Lambda)^{2}\right]}<1$, by Lemma 2.6 and taking the limit superior of both sides of inequality (20), we get

$$
\begin{aligned}
\limsup _{t \rightarrow+\infty} \frac{\ln I(t)}{t} & \leq\left[\frac{\lambda^{2}}{2 \sigma_{1}^{2}}-\frac{d^{2} \sigma_{2}^{2}}{2(d+\alpha \Lambda)^{2}}-\frac{d \beta}{d+\alpha \Lambda}-(d+r)\right] \\
& =\left(\frac{d \beta}{d+\alpha \Lambda}+d+r\right)\left(R_{3}-1\right)<0,
\end{aligned}
$$

which implies $\lim _{t \rightarrow+\infty} I(t)=0$.

Case (iv): When both $\lambda \geq \sigma_{1}^{2}$ and $R_{4}=\frac{2 \lambda}{\sigma_{1}^{2}+2(d+r)}+\frac{\beta^{2}}{\sigma_{2}^{2}\left[\sigma_{1}^{2}+2(d+r)\right]}<1$ hold, since $\lambda \geq \sigma_{1}^{2}$, the variable $S(t)$ in inequality (13) takes its maximum value on the interval $\left[0, \frac{\Lambda}{d}\right]$ at $\frac{\Lambda}{d}$, thus one has

$$
\begin{aligned}
\mathrm{d} \ln I(t) \leq & {\left[-\frac{d^{2} \sigma_{1}^{2}}{2 \Lambda^{2}}\left(\frac{\Lambda}{d}-\frac{\lambda \Lambda}{d \sigma_{1}^{2}}\right)^{2}-\frac{\sigma_{2}^{2}}{2}\left(\frac{1}{1+\alpha I(t)}+\frac{\beta}{\sigma_{2}^{2}}\right)^{2}+\frac{\lambda^{2}}{2 \sigma_{1}^{2}}+\frac{\beta^{2}}{2 \sigma_{2}^{2}}-(d+r)\right] \mathrm{d} t } \\
& +\frac{d \sigma_{1} S(t)}{\Lambda} \mathrm{d} B_{1}(t)-\frac{\sigma_{2}}{1+\alpha I(t)} \mathrm{d} B_{2}(t) \\
= & {\left[-\frac{\sigma_{1}^{2}}{2}\left(1+\frac{\lambda^{2}}{\sigma_{1}^{4}}-\frac{2 \lambda}{\sigma_{1}^{2}}\right)-\frac{\sigma_{2}^{2}}{2}\left(\frac{1}{1+\alpha I(t)}+\frac{\beta}{\sigma_{2}^{2}}\right)^{2}+\frac{\lambda^{2}}{2 \sigma_{1}^{2}}+\frac{\beta^{2}}{2 \sigma_{2}^{2}}-(d+r)\right] \mathrm{d} t } \\
& +\frac{d \sigma_{1} S(t)}{\Lambda} \mathrm{d} B_{1}(t)-\frac{\sigma_{2}}{1+\alpha I(t)} \mathrm{d} B_{2}(t) \\
= & {\left[-\frac{\sigma_{1}^{2}}{2}+\lambda-\frac{\sigma_{2}^{2}}{2}\left(\frac{1}{1+\alpha I(t)}+\frac{\beta}{\sigma_{2}^{2}}\right)^{2}+\frac{\beta^{2}}{2 \sigma_{2}^{2}}-(d+r)\right] \mathrm{d} t } \\
& +\frac{d \sigma_{1} S(t)}{\Lambda} \mathrm{d} B_{1}(t)-\frac{\sigma_{2}}{1+\alpha I(t)} \mathrm{d} B_{2}(t) \\
\leq & \left(-\frac{\sigma_{1}^{2}}{2}+\lambda+\frac{\beta^{2}}{2 \sigma_{2}^{2}}-(d+r)\right) \mathrm{d} t+\frac{d \sigma_{1} S(t)}{\Lambda} \mathrm{d} B_{1}(t)-\frac{\sigma_{2}}{1+\alpha I(t)} \mathrm{d} B_{2}(t) .
\end{aligned}
$$

Integrating both sides of inequality (21) from 0 to $t$ and dividing both sides by $t$ yield

$$
\frac{\ln I(t)}{t} \leq\left(-\frac{\sigma_{1}^{2}}{2}+\lambda+\frac{\beta^{2}}{2 \sigma_{2}^{2}}-(d+r)\right)+\frac{M_{1}(t)}{t}-\frac{M_{2}(t)}{t}+\frac{\ln I(0)}{t} .
$$


Applying the condition $R_{4}=\frac{2 \lambda}{\sigma_{1}^{2}+2(d+r)}+\frac{\beta^{2}}{\sigma_{2}^{2}\left[\sigma_{1}^{2}+2(d+r)\right]}<1$, by Lemma 2.6 and taking the limit superior of both sides of inequality (22), we get

$$
\begin{aligned}
\limsup _{t \rightarrow+\infty} \frac{\ln I(t)}{t} & \leq\left(-\frac{\sigma_{1}^{2}}{2}+\lambda+\frac{\beta^{2}}{2 \sigma_{2}^{2}}-(d+r)\right) \\
& =\left(d+r+\frac{\sigma_{1}^{2}}{2}\right)\left(R_{4}-1\right)<0,
\end{aligned}
$$

which implies $\lim _{t \rightarrow+\infty} I(t)=0$.

Since $\lim _{t \rightarrow+\infty} I(t)=0$, we have assumed the population size has reached limiting value. Further we consider the limit system of stochastic differential system (7)

$$
\mathrm{d} S(t)=\left[\Lambda-d S(t)+v\left(\frac{\Lambda}{d}-S(t)\right)\right] \mathrm{d} t .
$$

It is easy to see that $\lim _{t \rightarrow+\infty} S(t)=\frac{\Lambda}{d}$. This completes the proof of Theorem 2.1.

\subsection{Persistence in mean}

The conditions of the extinction for system (7) have been obtained. In this subsection, we investigate the conditions which lead to the persistence in mean of epidemic system (7) under the stochastic disturbances, which implies the infectious disease is prevalent.

Theorem 2.2 Let $(S(t), I(t))$ be the solution of system (7) with any initial value $(S(0), I(0)) \in$ $\mathbb{R}_{+}^{2}$. When both the conditions $\frac{d \lambda}{\alpha \Lambda} \geq d+v$ and

$$
\widetilde{R}=\frac{\alpha \Lambda(d+v)\left[2 \lambda-2 d-2 r-\left(\sigma_{1}^{2}+\sigma_{2}^{2}\right)\right]}{2 d \beta \lambda}>1
$$

hold, then the epidemic disease $I(t)$ is persistent in mean; in other words,

$$
0<\frac{\beta}{\alpha(v+d+r)}(\widetilde{R}-1) \leq \liminf _{t \rightarrow+\infty}|I(t)\rangle \leq \limsup _{t \rightarrow+\infty}\langle I(t)\rangle \leq \frac{\Lambda}{d} .
$$

Proof For system (7), computing the sum of two equations yields

$$
\begin{aligned}
\mathrm{d}(S(t)+I(t))= & {\left[\Lambda-d S(t)+\frac{v \Lambda}{d}-v S(t)-v I(t)-(d+r) I(t)-\frac{\beta I(t)}{1+\alpha I(t)}\right] \mathrm{d} t } \\
& -\frac{\sigma_{2} I(t)}{1+\alpha I(t)} \mathrm{d} B_{2}(t) \\
\geq & {\left[\Lambda+\frac{v \Lambda}{d}-(d+v) S(t)-(v+d+r) I(t)-\frac{\beta}{\alpha}\right] \mathrm{d} t } \\
& -\frac{\sigma_{2} I(t)}{1+\alpha I(t)} \mathrm{d} B_{2}(t) .
\end{aligned}
$$

Integrating both sides of inequality (23) from 0 to $t$ and dividing both sides by $t$, we obtain

$$
\begin{aligned}
\frac{1}{t}[S(t)-S(0)+I(t)-I(0)] \geq & \left(\Lambda+\frac{v \Lambda}{d}-\frac{\beta}{\alpha}\right)-(d+v)\langle S(t)\rangle-(v+d+r)\langle I(t)\rangle \\
& -\frac{M_{3}(t)}{t}
\end{aligned}
$$

where $M_{3}(t)=\int_{0}^{t} \frac{\sigma_{2} I(\theta)}{1+\alpha I(\theta)} \mathrm{d} B_{2}(\theta)$. 
Taking the limit of both sides of inequality (24) and by Lemma 2.6, we can get

$$
0 \geq\left(\Lambda+\frac{v \Lambda}{d}-\frac{\beta}{\alpha}\right)-(d+v) \lim _{t \rightarrow+\infty}\langle S(t)\rangle-(v+d+r) \lim _{t \rightarrow+\infty}\langle I(t)\rangle
$$

i.e.,

$$
\lim _{t \rightarrow+\infty}\langle S(t)\rangle \geq\left(\frac{\Lambda}{d}-\frac{\beta}{\alpha(d+v)}\right)-\frac{v+d+r}{d+v} \lim _{t \rightarrow+\infty}\langle I(t)\rangle .
$$

Next, we define a $C^{2}$-function $V: \mathbb{R}_{+}^{2} \rightarrow \mathbb{R}_{+}$by

$$
V(S(t), I(t))=\frac{1}{\alpha} \ln I(t)+I(t)+S(t) .
$$

Applying Itô's formula results in

$$
\mathrm{d} V(S(t), I(t))=\mathcal{L} V(S(t), I(t)) \mathrm{d} t+\frac{d \sigma_{1} S(t)}{\alpha \Lambda} \mathrm{d} B_{1}(t)-\frac{\sigma_{2}}{\alpha} \mathrm{d} B_{2}(t),
$$

where

$$
\begin{aligned}
\mathcal{L} V(S(t), I(t))= & {\left[\Lambda-d S(t)-\frac{d \lambda S(t) I(t)}{\Lambda}+v\left(\frac{\Lambda}{d}-S(t)-I(t)\right)\right] } \\
& +\frac{1+\alpha I(t)}{\alpha}\left[\frac{d \lambda S(t)}{\Lambda}-(d+r)-\frac{\beta}{1+\alpha I(t)}\right] \\
& -\frac{d^{2} \sigma_{1}^{2} S^{2}(t)}{2 \alpha \Lambda^{2}}-\frac{\sigma_{2}^{2}}{2 \alpha(1+\alpha I(t))^{2}} \\
= & \left(\Lambda+\frac{v \Lambda}{d}-\frac{d+r+\beta}{\alpha}\right)+\left(\frac{d \lambda}{\alpha \Lambda}-d-v\right) S(t) \\
& -(v+d+r) I(t)-\frac{d^{2} \sigma_{1}^{2} S^{2}(t)}{2 \alpha \Lambda^{2}}-\frac{\sigma_{2}^{2}}{2 \alpha(1+\alpha I(t))^{2}} \\
\geq & \left(\Lambda+\frac{v \Lambda}{d}-\frac{d+r+\beta}{\alpha}\right)+\left(\frac{d \lambda}{\alpha \Lambda}-d-v\right) S(t) \\
& -(v+d+r) I(t)-\frac{\sigma_{1}^{2}}{2 \alpha}-\frac{\sigma_{2}^{2}}{2 \alpha} \\
= & \left(\Lambda+\frac{v \Lambda}{d}-\frac{d+r+\beta}{\alpha}-\frac{\sigma_{1}^{2}+\sigma_{2}^{2}}{2 \alpha}\right)+\left(\frac{d \lambda}{\alpha \Lambda}-d-v\right) S(t) \\
& -(v+d+r) I(t) .
\end{aligned}
$$

Integrating both sides of inequality (26) from 0 to $t$ and dividing both sides by $t$, we get

$$
\begin{aligned}
\frac{1}{t}\left(\frac{1}{\alpha} \ln I(t)+I(t)+S(t)\right) \geq & \left(\Lambda+\frac{v \Lambda}{d}-\frac{d+r+\beta}{\alpha}-\frac{\sigma_{1}^{2}+\sigma_{2}^{2}}{2 \alpha}\right)+\left(\frac{d \lambda}{\alpha \Lambda}-d-v\right)\langle S(t)\rangle \\
& -(v+d+r)\langle I(t)\rangle+\frac{M_{1}(t)}{\alpha t}-\frac{M_{4}(t)}{t} \\
& +\frac{1}{t}\left(\frac{1}{\alpha} \ln I(0)+I(0)+S(0)\right)
\end{aligned}
$$


i.e.,

$$
\begin{aligned}
(v+d+r)\langle I(t)\rangle \geq & \left(\Lambda+\frac{v \Lambda}{d}-\frac{d+r+\beta}{\alpha}-\frac{\sigma_{1}^{2}+\sigma_{2}^{2}}{2 \alpha}\right)+\left(\frac{d \lambda}{\alpha \Lambda}-d-v\right)\langle S(t)\rangle+\frac{M_{1}(t)}{\alpha t} \\
& -\frac{M_{4}(t)}{t}-\frac{\ln I(t)-\ln I(0)}{\alpha t}-\frac{I(t)-I(0)}{t}-\frac{S(t)-S(0)}{t} \\
\geq & \left\{\begin{array}{cc}
\left(\Lambda+\frac{v \Lambda}{d}-\frac{d+r+\beta}{\alpha}-\frac{\sigma_{1}^{2}+\sigma_{2}^{2}}{2 \alpha}\right)+\left(\frac{d \lambda}{\alpha \Lambda}-d-v\right)\langle S(t)\rangle+\frac{M_{1}(t)}{\alpha t} \\
-\frac{M_{4}(t)}{t}+\frac{\ln I(0)}{\alpha t}-\frac{I(t)-I(0)}{t}-\frac{S(t)-S(0)}{t}, \quad 0<I(t)<1 \\
\left(\Lambda+\frac{v \Lambda}{d}-\frac{d+r+\beta}{\alpha}-\frac{\sigma_{1}^{2}+\sigma_{2}^{2}}{2 \alpha}\right)+\left(\frac{d \lambda}{\alpha \Lambda}-d-v\right)\langle S(t)\rangle+\frac{M_{1}(t)}{\alpha t} \\
-\frac{M_{4}(t)}{t}-\frac{\ln I(t)-\ln I(0)}{\alpha t}-\frac{I(t)-I(0)}{t}-\frac{S(t)-S(0)}{t}, \quad 1 \leq I(t),
\end{array}\right.
\end{aligned}
$$

where $M_{1}(t)=\int_{0}^{t} \frac{d \sigma_{1} S(\theta)}{\Lambda} \mathrm{d} B_{1}(\theta)$ and $M_{4}(t)=\int_{0}^{t} \frac{\sigma_{2}}{\alpha} \mathrm{d} B_{2}(\theta)$.

Taking the limit of both sides of inequality (27), by Lemma 2.6, we have

$$
\begin{aligned}
(v+d+r) \lim _{t \rightarrow+\infty}\langle I(t)\rangle \geq & \left(\Lambda+\frac{v \Lambda}{d}-\frac{d+r+\beta}{\alpha}-\frac{\sigma_{1}^{2}+\sigma_{2}^{2}}{2 \alpha}\right) \\
& +\left(\frac{d \lambda}{\alpha \Lambda}-d-v\right) \lim _{t \rightarrow+\infty}\langle S(t)\rangle .
\end{aligned}
$$

From inequality (25) and inequality (28), and assuming that the condition $\frac{d \lambda}{\alpha \Lambda} \geq d+v$ holds, then we can obtain

$$
\begin{aligned}
(v+ & d+r) \lim _{t \rightarrow+\infty}\langle I(t)\rangle \\
\geq & \left(\Lambda+\frac{v \Lambda}{d}-\frac{d+r+\beta}{\alpha}-\frac{\sigma_{1}^{2}+\sigma_{2}^{2}}{2 \alpha}\right) \\
& +\left(\frac{d \lambda}{\alpha \Lambda}-d-v\right)\left[\left(\frac{\Lambda}{d}-\frac{\beta}{\alpha(d+v)}\right)-\frac{v+d+r}{d+v} \lim _{t \rightarrow+\infty}\langle I(t)\rangle\right] \\
= & +\frac{v \Lambda}{d}-\frac{d+r+\beta}{\alpha}-\frac{\sigma_{1}^{2}+\sigma_{2}^{2}}{2 \alpha}+\frac{\lambda}{\alpha}-\frac{\lambda d \beta}{\alpha^{2} \Lambda(d+v)}-\frac{(d+v) \Lambda}{d}+\frac{\beta}{\alpha} \\
& -\left[\frac{\lambda d(v+d+r)}{\alpha \Lambda(d+v)}-(v+d+r)\right] \lim _{t \rightarrow+\infty}\langle I(t)\rangle,
\end{aligned}
$$

i.e.,

$$
\begin{aligned}
\lim _{t \rightarrow+\infty}\langle I(t)\rangle & \geq\left[\frac{2 \lambda-2 d-2 r-\left(\sigma_{1}^{2}+\sigma_{2}^{2}\right)}{2 \alpha}-\frac{\lambda d \beta}{\alpha^{2} \Lambda(d+v)}\right] \cdot \frac{\alpha \Lambda(d+v)}{\lambda d(v+d+d r)} \\
& =\frac{\beta}{\alpha(v+d+r)}\left[\frac{\alpha \Lambda(d+v)\left[2 \lambda-2 d-2 r-\left(\sigma_{1}^{2}+\sigma_{2}^{2}\right)\right]}{2 d \beta \lambda}-1\right] \\
& :=\frac{\beta}{\alpha(v+d+r)}(\widetilde{R}-1) .
\end{aligned}
$$

When the condition $\widetilde{R}=\frac{\alpha \Lambda(d+v)\left[2 \lambda-2 d-2 r-\left(\sigma_{1}^{2}+\sigma_{2}^{2}\right)\right]}{2 d \beta \lambda}>1$ holds, taking the inferior limit of both sides of (29) yields

$$
\liminf _{t \rightarrow+\infty}\langle I(t)\rangle \geq \frac{\beta}{\alpha(v+d+r)}(\widetilde{R}-1)>0 .
$$


By Lemma 2.4, we know limsup $\sup _{t \rightarrow+\infty} I(t) \leq \frac{\Lambda}{d}$, then $\lim \sup _{t \rightarrow+\infty}\langle I(t)\rangle \leq \frac{\Lambda}{d}$. Therefore, we get

$$
0<\frac{\beta}{\alpha(v+d+r)}(\widetilde{R}-1) \leq \liminf _{t \rightarrow+\infty}\langle I(t)\rangle \leq \limsup _{t \rightarrow+\infty}\langle I(t)\rangle \leq \frac{\Lambda}{d} .
$$

This completes the proof of Theorem 2.2.

\section{Conclusions and numerical simulations}

In this paper, we investigate the stochastic SIRS epidemic model with standard incidence rate and saturated treatment function. We first prove the existence and uniqueness of a global positive solution for the corresponding limiting system (7). Then we investigate the persistence in mean and extinction of the stochastic SIRS epidemic system by using the Lyapunov method and the techniques of a series of stochastic inequalities. The biological significance of our results shows that the ability of the epidemic model to adapt to the external environment disturbance is limited, and the external environment disturbances may have important effect on the stability of the SIRS epidemic system. When the perturbations in the environment are small enough, the stability of the stochastic SIRS epidemic system cannot be destroyed, while large disturbances occurring in the environment can lead to the extinction of epidemic diseases. Therefore, this shows that the environmental distributions are advantageous to controlling infectious diseases. Our results significantly improve and generalize the corresponding results in recent literature. The developed theoretical methods and stochastic inequalities techniques can be applied to explore the highdimensional nonlinear stochastic differential systems.

Next, we give some numerical simulations to illustrate our theoretical results. The discrete equations of system (7) are described by

$$
\begin{aligned}
S_{n+1}=S_{n}+ & {\left[\Lambda-d S_{n}-\frac{d \lambda S_{n} I_{n}}{\Lambda}+v\left(\frac{\Lambda}{d}-S_{n}-I_{n}\right)\right] \Delta t } \\
& -\sigma_{1} \frac{d S_{n} I_{n}}{\Lambda} \sqrt{\Delta t} W_{1 n}-\frac{\sigma_{1}^{2}}{2} \frac{d S_{n} I_{n}}{\Lambda}\left(W_{1 n}^{2}-1\right) \Delta t, \\
I_{n+1}=I_{n}+ & {\left[\frac{d \lambda S_{n} I_{n}}{\Lambda}-(d+r) I_{n}-\frac{\beta I_{n}}{1+\alpha I_{n}}\right] \Delta t } \\
& +\sigma_{1} \frac{d S_{n} I_{n}}{\Lambda} \sqrt{\Delta t} W_{1 n}+\frac{\sigma_{1}^{2}}{2} \frac{d S_{n} I_{n}}{\Lambda}\left(W_{1 n}^{2}-1\right) \Delta t \\
& -\sigma_{2} \frac{I_{n}}{1+\alpha I_{n}} \sqrt{\Delta t} W_{2 n}-\frac{\sigma_{2}^{2}}{2} \frac{I_{n}}{1+\alpha I_{n}}\left(W_{2 n}^{2}-1\right) \Delta t,
\end{aligned}
$$

where $W_{1 n}, W_{2 n}, n=1,2, \ldots$, are independent Gaussian random variables $N(0,1)$. Here, we let $\Delta t=0.01$.

In the following figures, we let $S(0)=0.1, I(0)=0.1, \alpha=0.3, \beta=0.01, \lambda=0.7, d=0.5$, $\Lambda=0.1, r=0.07, v=0.5$, and the step size $\Delta t=0.01$.

Figure 1(a) is the deterministic model of stochastic system (7) with $\sigma_{1}=\sigma_{2}=0$. Figure 1 (b) shows the stochastic epidemic system (7) with $\sigma_{1}=0.7$ and $\sigma_{2}=0.1$. By computation, we get that

$$
R_{1}=\frac{\lambda^{2}}{2(d+r) \sigma_{1}^{2}}+\frac{\beta^{2}}{2(d+r) \sigma_{2}^{2}}=0.886<1, \quad \lim _{t \rightarrow+\infty} S(t)=\frac{\Lambda}{d}=0.2, \quad \lim _{t \rightarrow+\infty} I(t)=0,
$$



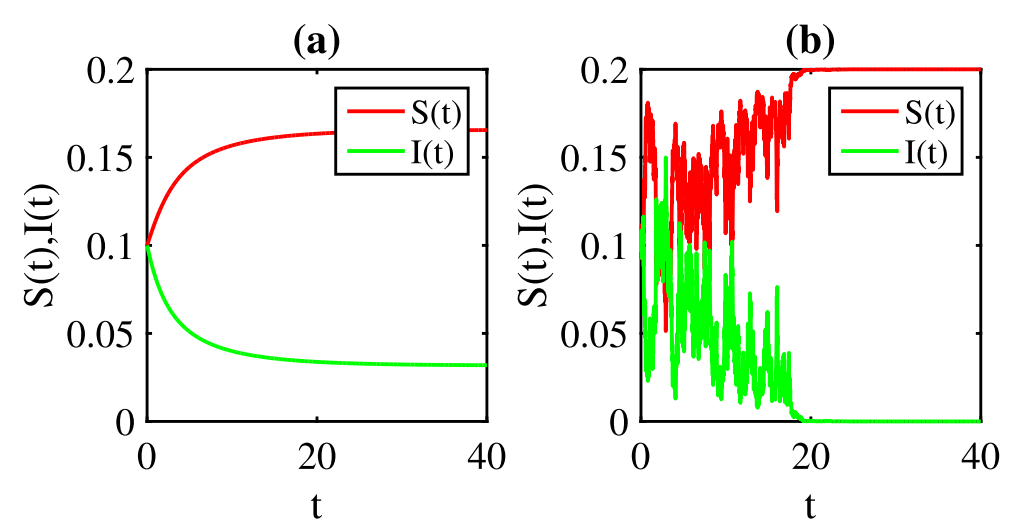

Figure 1 Time sequence diagram: (a) the deterministic epidemic model, (b) the stochastic epidemic system (7) for case (i) of Theorem 2.1
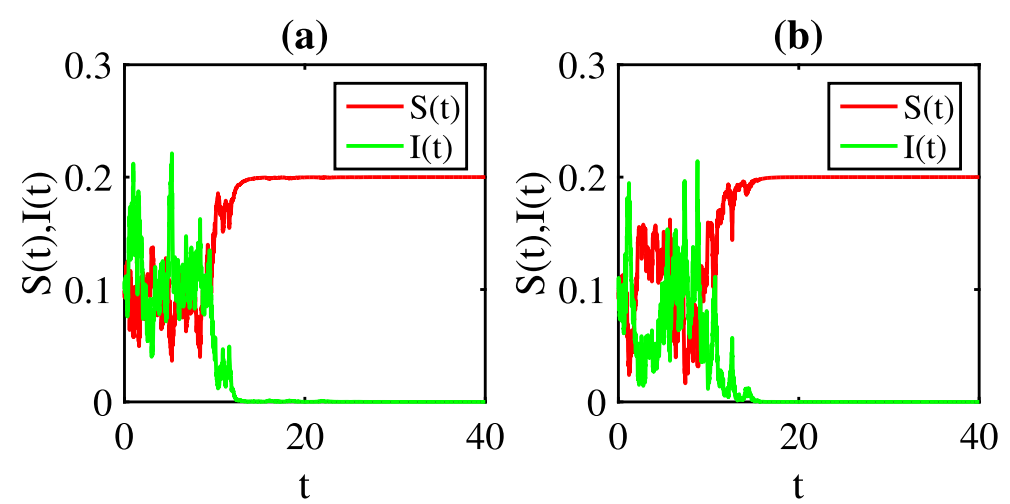

Figure 2 Time sequence diagram of stochastic system (7): (a) case (ii) of Theorem 2.1, (b) case (iii) of Theorem 2.1

which satisfy case (i) of Theorem 2.1. For Figure 2(a) with $\sigma_{1}=0.5$ and $\sigma_{2}=0.2$, we get that

$$
\begin{aligned}
& \lambda=0.7 \geq 0.25=0.5^{2}=\sigma_{1}^{2}, \\
& R_{2}=\frac{2(\lambda-d-r)(d+\alpha \Lambda)^{2}}{\sigma_{1}^{2}(d+\alpha \Lambda)^{2}+d^{2} \sigma_{2}^{2}}-\frac{2 d \beta(d+\alpha \Lambda)}{\sigma_{1}^{2}(d+\alpha \Lambda)^{2}+d^{2} \sigma_{2}^{2}}=0.8443<1,
\end{aligned}
$$

and

$$
\lim _{t \rightarrow+\infty} S(t)=\frac{\Lambda}{d}=0.2, \quad \lim _{t \rightarrow+\infty} I(t)=0,
$$

which satisfy case (ii) of Theorem 2.1. For Figure 2(b) with $\sigma_{1}=0.85$ and $\sigma_{2}=0.3$, we get that

$$
\begin{aligned}
& \lambda=0.7<0.7225=0.85^{2}=\sigma_{1}^{2}, \\
& R_{3}=\frac{\lambda^{2}(d+\alpha \Lambda)}{2 \sigma_{1}^{2}[d \beta+(d+r)(d+\alpha \Lambda)]}-\frac{d^{2} \sigma_{2}^{2}}{2\left[d \beta(d+\alpha \Lambda)+(d+r)(d+\alpha \Lambda)^{2}\right]}=0.5161<1,
\end{aligned}
$$



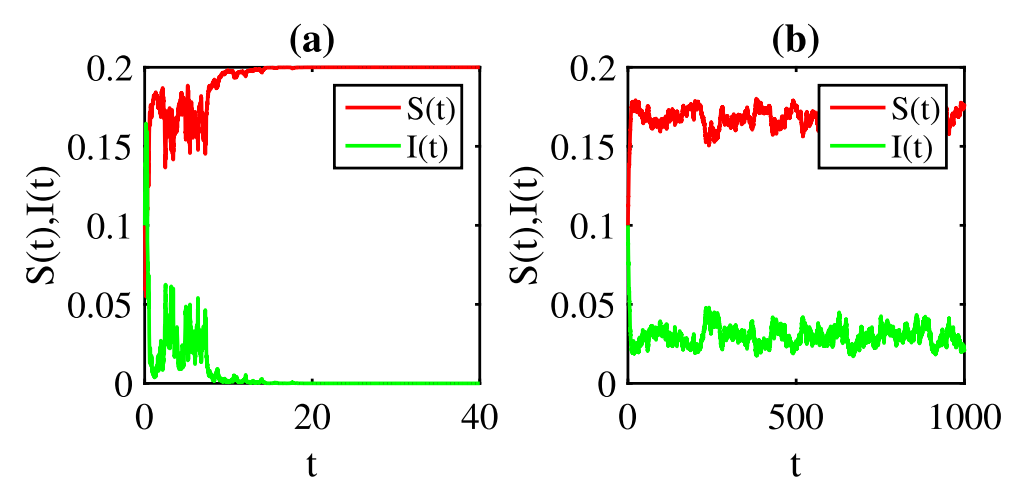

Figure 3 Time sequence diagram of stochastic system (7): (a) case (iv) of Theorem 2.1, (b) case of Theorem 2.2

and

$$
\lim _{t \rightarrow+\infty} S(t)=\frac{\Lambda}{d}=0.2, \quad \lim _{t \rightarrow+\infty} I(t)=0,
$$

which satisfy case (iii) of Theorem 2.1. For Figure 3(a) with $\sigma_{1}=0.7$ and $\sigma_{2}=0.3$, we get that

$$
\lambda=0.7 \geq 0.49=0.7^{2}=\sigma_{1}^{2}, \quad R_{4}=\frac{2 \lambda}{\sigma_{1}^{2}+2(d+r)}+\frac{\beta^{2}}{\sigma_{2}^{2}\left[\sigma_{1}^{2}+2(d+r)\right]}=0.85958<1,
$$

and

$$
\lim _{t \rightarrow+\infty} S(t)=\frac{\Lambda}{d}=0.2, \quad \lim _{t \rightarrow+\infty} I(t)=0,
$$

which satisfy case (iv) of Theorem 2.1 .

According to Figures 1(b), 2(a), 2(b) and 3(a), the disease $I(t)$ in the stochastic epidemic system (7) is extinct. Comparing Figure 1(a) with Figures 1(b), 2(a), 2(b) and 3(a), we can see that when the environmental fluctuations are large enough, they can lead to the extinction of disease. Thus, the random fluctuations are beneficial to the control of epidemic diseases. This is consistent with our conclusion in Theorem 2.1.

For Figure 3(b), we let $\sigma_{1}=\sigma_{2}=0.03$. By computation, we get that

$$
\frac{d \lambda}{\alpha \Lambda}=11.6 \geq 1=d+v, \quad \widetilde{R}=\frac{\alpha \Lambda(d+v)\left[2 \lambda-2 d-2 r-\left(\sigma_{1}^{2}+\sigma_{2}^{2}\right)\right]}{2 d \beta \lambda}=1.10657>1,
$$

which satisfies the conditions of Theorem 2.2. Comparing Figure 1(a) with Figure 3(b), when the white noises are small, the stochastic epidemic system (7) is persistent in mean. This is consistent with our conclusion in Theorem 2.2.

Obviously, the numerical simulation results are consistent with the conclusions of our theorems.

To sum up, our main results are summarized as follows:

I. Extinction

When one of the following conditions holds:

(i) $R_{1}=\frac{\lambda^{2}}{2(d+r) \sigma_{1}^{2}}+\frac{\beta^{2}}{2(d+r) \sigma_{2}^{2}}<1$, 
(ii) $\lambda \geq \sigma_{1}^{2}$ and $R_{2}=\frac{2(\lambda-d-r)(d+\alpha \Lambda)^{2}}{\sigma_{1}^{2}(d+\alpha \Lambda)^{2}+d^{2} \sigma_{2}^{2}}-\frac{2 d \beta(d+\alpha \Lambda)}{\sigma_{1}^{2}(d+\alpha \Lambda)^{2}+d^{2} \sigma_{2}^{2}}<1$,

(iii) $\lambda<\sigma_{1}^{2}$ and $R_{3}=\frac{\lambda^{2}(d+\alpha \Lambda)}{2 \sigma_{1}^{2}[d \beta+(d+r)(d+\alpha \Lambda)]}-\frac{d^{2} \sigma_{2}^{2}}{2\left[d \beta(d+\alpha \Lambda)+(d+r)(d+\alpha \Lambda)^{2}\right]}<1$,

(iv) $\lambda \geq \sigma_{1}^{2}$ and $R_{4}=\frac{2 \lambda}{\sigma_{1}^{2}+2(d+r)}+\frac{\beta^{2}}{\sigma_{2}^{2}\left[\sigma_{1}^{2}+2(d+r)\right]}<1$,

then

$$
\lim _{t \rightarrow+\infty} S(t)=\frac{\Lambda}{d}, \quad \lim _{t \rightarrow+\infty} I(t)=0
$$

\section{Persistence in mean}

When both conditions $\frac{d \lambda}{\alpha \Lambda} \geq d+v$ and

$$
\widetilde{R}=\frac{\alpha \Lambda(d+v)\left[2 \lambda-2 d-2 r-\left(\sigma_{1}^{2}+\sigma_{2}^{2}\right)\right]}{2 d \beta \lambda}>1
$$

hold, then the epidemic disease $I(t)$ is persistent in mean; in other words,

$$
0<\frac{\beta}{\alpha(v+d+r)}(\widetilde{R}-1) \leq \liminf _{t \rightarrow+\infty}\langle I(t)\rangle \leq \limsup _{t \rightarrow+\infty}\langle I(t)\rangle \leq \frac{\Lambda}{d}
$$

\section{Acknowledgements}

This research was partially supported by the National Natural Science Foundation of China $(11371230,11501331$, 11561004), by Joint Innovative Center for Safe and Effective Mining Technology and Equipment of Coal Resources, the SDUST Research Fund (2014TDJH102), by the Open Foundation of the Key Laboratory of Jiangxi Province for Numerical Simulation and Emulation Techniques, Gannan Normal University, China, and by Shandong Provincial Natural Science Foundation, China (ZR2015AQ001, BS2015SF002).

\section{Competing interests}

The authors declare that they have no competing interests.

\section{Authors' contributions}

The work presented in this paper has been accomplished through contributions of all authors. All authors read and approved the final manuscript.

\section{Author details}

${ }^{1}$ College of Mathematics and Systems Science, Shandong University of Science and Technology, Qingdao, P.R. China. ${ }^{2}$ State Key Laboratory of Mining Disaster Prevention and Control Co-founded by Shandong Province and the Ministry of Science and Technology, Shandong University of Science and Technology, Qingdao, P.R. China. ${ }^{3}$ Open Foundation of the Key Laboratory of Jiangxi Province for Numerical Simulation and Emulation Techniques, Gannan Normal University, Ganzhou, China.

\section{Publisher's Note}

Springer Nature remains neutral with regard to jurisdictional claims in published maps and institutional affiliations.

Received: 13 November 2017 Accepted: 28 January 2018 Published online: 05 February 2018

\section{References}

1. Lakshmikantham, V., Vatsala, A.S.: Theory of Differential and Integral Inequalities with Initial Time Difference and Applications. Springer, Berlin (1999)

2. Jleli, M., Kirane, M., Samet, B.: Lyapunov-type inequalities for fractional partial differential equations. Appl. Math. Lett. 66, 30-39 (2017)

3. Bai, Z.B., Zhang, S., Sun, S.J., Yin, C.: Monotone iterative method for fractional differential equations. Electron. J. Differ. Equ. 2016, 6 (2016)

4. Ma, Q.H., Ma, C.H.A.O., Wang, J.X.: A Lyapunov-type inequality for a fractional differential equation with Hadamard derivative. J. Math. Inequal. 11, 135-141 (2017)

5. Cui, Y.J.: Uniqueness of solution for boundary value problems for fractional differential equations. Appl. Math. Lett. 51, 48-54 (2016)

6. Zhang, T.Q., Ma, W.B., Meng, X.Z.: Impulsive control of a continuous-culture and flocculation harvest chemostat model. Int. J. Syst. Sci. 2017, 3459-3469 (2017)

7. Bai, Z.B., Dong, X.Y., Yin, C.: Existence results for impulsive nonlinear fractional differential equation with mixed boundary conditions. Bound. Value Probl. 2016, 63 (2016)

8. Zhang, T.Q., Meng, X.Z., Song, Y., Zhang, T.H.: A stage-structured predator-prey SI model with disease in the prey and impulsive effects. Math. Model. Anal. 18, 505-528 (2013) 
9. Li, Z.X., Chen, L.S., Liu, Z.J.: Periodic solution of a chemostat model with variable yield and impulsive state feedback control. Appl. Math. Model. 36(3), 1255-1266 (2012)

10. Cheng, H.D., Zhang, T.Q.: A new predator-prey model with a profitless delay of digestion and impulsive perturbation on the prey. Appl. Math. Comput. 217(22), 9198-9208 (2011)

11. Feng, T., Meng, X.Z., Liu, L.D., Gao, S.J.: Application of inequalities technique to dynamics analysis of a stochastic eco-epidemiology model. J. Inequal. Appl. 2016, 327 (2016)

12. Wang, Y., Jiang, D.Q., Hayat, T., Ahmad, B.: A stochastic HIV infection model with T-cell proliferation and CTL immune response. Appl. Math. Comput. 315, 477-493 (2017)

13. Zhao, Y.N., Jiang, D.Q., O'Regan, D.: The extinction and persistence of the stochastic SIS epidemic model with vaccination. Phys. A, Stat. Mech. Appl. 392, 4916-4927 (2013)

14. Miao, A.Q., Zhang, J., Zhang, T.Q., Sampath Aruna Pradeep, B.G.: Threshold dynamics of a stochastic SIR model with vertical transmission and vaccination. Comput. Math. Methods Med. 2017, 4820183 (2017)

15. Ma, H.J., Jia, Y.M.: Stability analysis for stochastic differential equations with infinite Markovian switchings. J. Math. Anal. Appl. 435(1), 593-605 (2016)

16. Zhang, Y., Chen, S.H., Gao, S.J.: Analysis of a nonautonomous stochastic predator-prey model with Crowley-Martin functional response. Adv. Differ. Equ. 2016, 264 (2016)

17. Zhang, S.Q., Meng, X.Z., Feng, T., Zhang, T.H.: Dynamics analysis and numerical simulations of a stochastic non-autonomous predator-prey system with impulsive effects. Nonlinear Anal. Hybrid Syst. 26, 19-37 (2017)

18. Liu, G.D., Wang, X.H., Meng, X.Z., Gao, S.J.: Extinction and persistence in mean of a novel delay impulsive stochastic infected predator-prey system with jumps. Complexity 2017, 1950970 (2017)

19. Liu, M., Du, C.X., Deng, M.L.: Persistence and extinction of a modified Leslie-Gower Holling-type II stochastic predator-prey model with impulsive toxicant input in polluted environments. Nonlinear Anal. Hybrid Syst. 27, 177-190 (2018)

20. Meng, X.Z., Wang, L., Zhang, T.H.: Global dynamics analysis of a nonlinear impulsive stochastic chemostat system in a polluted environment. J. Appl. Anal. Comput. 6, 865-875 (2016)

21. Li, C.H., Tsai, C.C., Yang, S.Y.: Analysis of epidemic spreading of an SIRS model in complex heterogeneous networks. Commun. Nonlinear Sci. Numer. Simul. 19, 1042-1054 (2014)

22. Yang, Q.S., Mao, X.R.: Extinction and recurrence of multi-group SEIR epidemic models with stochastic perturbations. Nonlinear Anal., Real World Appl. 14, 1434-1456 (2013)

23. Zhang, T.Q., Meng, X.Z., Zhang, T.H., Song, Y.: Global dynamics for a new high-dimensional SIR model with distributed delay. Appl. Math. Comput. 218, 11806-11819 (2012)

24. Meng, X.Z., Chen, L.S., Wu, B.: A delay SIR epidemic model with pulse vaccination and incubation times. Nonlinear Anal., Real World Appl. 11, 88-98 (2010)

25. Artalejo, J.R., Economou, A., Lopez-Herrero, M.J.: The stochastic SEIR model before extinction: computational approaches. Appl. Math. Comput. 265, 1026-1043 (2015)

26. Liu, W.M., Levin, S.A., Iwasa, Y.: Influence of nonlinear incidence rates upon the behavior of SIRS epidemiological models. J. Math. Biol. 23, 187-204 (1986)

27. Li, G.H., Wang, W.D.: Bifurcation analysis of an epidemic model with nonlinear incidence. Appl. Math. Comput. 214, $411-423(2009)$

28. Zhang, T.Q., Meng, X.Z., Zhang, T.H.: Global analysis for a delayed SIV model with direct and environmental transmissions. J. Appl. Anal. Comput. 6, 479-491 (2016)

29. Xiao, Y.N., Tang, S.Y.: Dynamics of infection with nonlinear incidence in a simple vaccination model. Nonlinear Anal, Real World Appl. 11, 4154-4163 (2010)

30. Zhao, D.L., Yuan, S.L.: Break-even concentration and periodic behavior of a stochastic chemostat model with seasonal fluctuation. Commun. Nonlinear Sci. Numer. Simul. 46, 62-73 (2017)

31. Zhang, X., Liu, X., Li, Y.: Adaptive fuzzy tracking control for nonlinear strict-feedback systems with unmodeled dynamics via backstepping technique. Neurocomputing 235, 182-191 (2017)

32. Mena-Lorcat, J., Hethcote, H.W.: Dynamic models of infectious diseases as regulators of population sizes. J. Math. Biol. 30, 693-716 (1992)

33. Hu, Z.X., Liu, S., Wang, H.: Backward bifurcation of an epidemic model with standard incidence rate and treatment rate. Nonlinear Anal., Real World Appl. 9, 2302-2312 (2008)

34. Safi, M.A., Gumel, A.B., Elbasha, E.H.: Qualitative analysis of an age-structured SEIR epidemic model with treatment. Appl. Math. Comput. 219, 10627-10642 (2013)

35. Zhou, L.H., Fan, M.: Dynamics of an SIR epidemic model with limited medical resources revisited. Nonlinear Anal., Real World Appl. 13, 312-324 (2012)

36. Wei, J.., Cui, J.A.: Dynamics of SIS epidemic model with the standard incidence rate and saturated treatment function. Int. J. Biomath. 5, 1260003 (2012)

37. Wang, W.D., Ruan, S.G.: Bifurcations in an epidemic model with constant removal rate of the infectives. J. Math. Anal. Appl. 291, 775-793 (2004)

38. Wang, W.D.: Backward bifurcation of an epidemic model with treatment. Math. Biosci. 201, 58-71 (2006)

39. Eckalbar, J.C., Eckalbar, W.L.: Dynamics of an epidemic model with quadratic treatment. Nonlinear Anal., Real World Appl. 12, 320-332 (2011)

40. Zhang, X., Liu, X.N.: Backward bifurcation of an epidemic model with saturated treatment function. J. Math. Anal. Appl. 348, 433-443 (2008)

41. Gao, Y.X., Zhang, W.P., Liu, D., Xiao, Y.J.: Bifurcation analysis of an SIRS epidemic model with standard incidence rate and saturated treatment function. J. Appl. Anal. Comput. 7, 1070-1094 (2017)

42. May, R.M.C.: Stability and Complexity in Model Ecosystems. Princeton University Press, Princeton (2001)

43. Liu, Q., Jiang, D.Q.: Stationary distribution and extinction of a stochastic SIR model with nonlinear perturbation. Appl. Math. Lett. 73, 8-15 (2017)

44. Liu, M., Fan, M.: Permanence of stochastic Lotka-Volterra systems. J. Nonlinear Sci. 27, 425-452 (2017)

45. Wang, W.M., Cai, Y.L., Li, J.L., Gui, Z.J.: Periodic behavior in a FIV model with seasonality as well as environment fluctuations. J. Franklin Inst. 354, 7410-7428 (2017) 
46. LV, X.J., Wang, L., Meng, X.Z.: Global analysis of a new nonlinear stochastic differential competition system with impulsive effect. Adv. Differ. Equ. 2017, 296 (2017)

47. Cai, Y.L., Kang, Y., Wang, W.M.: A stochastic SIRS epidemic model with nonlinear incidence rate. Appl. Math. Comput. $305,221-240(2017)$

48. Liu, L.D., Meng, X.Z:: Optimal harvesting control and dynamics of two species stochastic model with delays. Adv. Differ. Equ. 2017, 18 (2017)

49. Zhao, Y., Yuan, S.L.: Optimal harvesting policy of a stochastic two-species competitive model with Lévy noise in a polluted environment. Phys. A, Stat. Mech. Appl. 477, 20-33 (2017)

50. Wang, J.M., Cheng, H.D., Meng, X.Z., Pradeep, B.G.S.A.: Geometrical analysis and control optimization of a predator-prey model with multi state-dependent impulse. Adv. Differ. Equ. 2017, 252 (2017)

51. Cai, Y.L., Kang, Y., Banerjee, M., Wang, W.M.: Complex dynamics of a host-parasite model with both horizontal and vertical transmissions in a spatial heterogeneous environment. Nonlinear Anal., Real World Appl. 40, 444-465 (2018)

52. Miao, A.Q., Wang, X.Y., Zhang, T.Q., Wang, W., Sampath Aruna Pradeep, B.G.: Dynamical analysis of a stochastic SIS epidemic model with nonlinear incidence rate and double epidemic hypothesis. Adv. Differ. Equ. 2017, 226 (2017)

53. Mao, X.R.: Stochastic Differential Equations and Applications. Elsevier, Amsterdam (2007)

54. Meng, X.Z., Zhao, S.N., Feng, T., Zhang, T.H.: Dynamics of a novel nonlinear stochastic SIS epidemic model with double epidemic hypothesis. J. Math. Anal. Appl. 433, 227-242 (2016)

\section{Submit your manuscript to a SpringerOpen ${ }^{\circ}$ journal and benefit from:}

- Convenient online submission

- Rigorous peer review

- Open access: articles freely available online

- High visibility within the field

- Retaining the copyright to your article

Submit your next manuscript at $\boldsymbol{\Delta}$ springeropen.com 